\title{
An Efficient Method for Aneurysm Volume Quantification Applicable in Any Shape and Modalities
}

\author{
Jaewoo Chung, Jung Ho Ko \\ Department of Neurosurgery, Dankook University College of Medicine, Cheonan, Korea
}

Objective : Aneurysm volume quantification (AVQ) using the equation of ellipsoid volume is widely used although it is inaccurate. Furthermore, AVQ with 3-dimensional (3D) rendered data has limitations in general use. A novel universal method for AVQ is introduced for any diagnostic modality and application to any shape of aneurysms.

Methods : Relevant AVQ studies published from January 1997 to June 2019 were identified to determine common methods of AVQ. The basic idea is to eliminate the normal artery volume from 3D model with the aneurysm. After Digital Imaging and Communications in Medicine (DICOM) data is converted and exported to stereolithography (STL) file format, the 3D STL model is modified to remove the aneurysm and the volume difference between the 3D model with/without the aneurysm is defined as the aneurysm volume. Fifty randomly selected aneurysms from DICOM database were used to validate the different AVQ methods.

Results : We reviewed and categorized AVQ methods in 121 studies. Approximately $60 \%$ used the ellipsoid method, while $24 \%$ used the 3D model. For 50 randomly selected aneurysms, volumes were measured using 3D Slicer, RadiAnt, and ellipsoid method. Using 3D Slicer as the reference, the ratios of mean difference to mean volume obtained by RadiAnt and ellipsoid method were $-1.21 \pm 7.46 \%$ and $4.04 \pm 30.54 \%$, respectively. The deviations between RadiAnt and 3D Slicer were small despite of aneurysm shapes, but those of ellipsoid method and 3D Slicer were large.

Conclusion : In spite of inaccuracy, ellipsoid method is still mostly used. We propose a novel universal method for AVQ that is valid, low cost, and easy to use.

Key Words : Intracranial aneurysm · Volume quantification · Ellipsoid method · 3D model.

\section{INTRODUCTION}

The coil volume embolization ratio (VER) was first introduced in 1997 by Satoh et al. ${ }^{27}$. The authors considered an aneurysm as an ellipsoid and used the mathematical equation for ellipsoid volume to calculate aneurysm volume. However, this method of aneurysm volume quantification (AVQ) may contain errors and is not compatible for use with irregularly shaped or fusiform aneurysms $\mathrm{s}^{26,32)}$. Nonetheless, it has been widely used in studies and applied to commercial software for calculating coil packing density. Other AVQ methods have been introduced and improved upon with 3-dimensional (3D) angiographic data, but these also have limitations related to imaging modality, angiography equipment (workstations), and the particular software utilized ${ }^{2,5,6,8,9,23,26}$. Therefore, the applicability of these methods to clinical use and research is

- Received : August 31, 2020 •Revised : November 23, 2020 •Accepted : December 14, 2020

- Address for reprints : Jaewoo Chung

Department of Neurosurgery, Dankook University College of Medicine, 201 Manghyang-ro, Dongnam-gu, Cheonan 31116, Korea Tel : +82-41-550-3034, Fax : +82-504-219-7964, E-mail : jchung@dkuh.co.kr, ORCID : https://orcid.org/0000-0002-3512-6610 
also somewhat limited. In order to overcome these problems, we introduce a novel universal method for AVQ that can be used with any diagnostic modality and is applicable to any aneurysm shape.

\section{MATERIALS AND METHODS}

This retrospective study was approved by the Institutional Review Board of Dankook University Hospital (2020-07-017) before data collection began. Because of the retrospective design of the study the patient consent for this study was waived by the Institutional Review Board.

\section{Literature review}

The PubMed database was searched using the keywords "VER", "packing density", and "aneurysm volume" to identify relevant AVQ studies published between January 1997 and June 2019. Each study was reviewed and categorized according to AVQ method and year of publication. When the method was not clearly described, we followed the reference cited in the manuscript's definition of aneurysm volume. Based on our review, we established the following AVQ categories : 2-dimensional (2D) assessment, ellipsoid method, AngioCalc and AngioSuite, Workstation, commercial software, and phantom. The 2D assessment category includes non-quantitative methods that use plain radiographic images such as the Raymond-Roy occlusion classification ${ }^{30}$. The ellipsoid method uses the mathematical equation for ellipsoid volume. The AngioCalc and Angiosuite category is comprised of the webbased and mobile device applications AngioCalc and AngioSuite Neuro Edition, respectively. In brief, they each use the ellipsoid method and automatically calculate coil volume and VER. Workstation is software provided for angiography suite equipment. The commercial software category is comprised of various types of mathematical, computer-aided design (CAD), and computational fluid dynamics (CFD) software. The phantom category is comprised of experimental methods that use phantom models for AVQ.

\section{Definition of aneurysm volume}

Aneurysm volume is usually defined as the volume of the outpouching abnormal vascular lesion above the aneurysm neck as in Fig. 1A. However, determination of the aneurysm

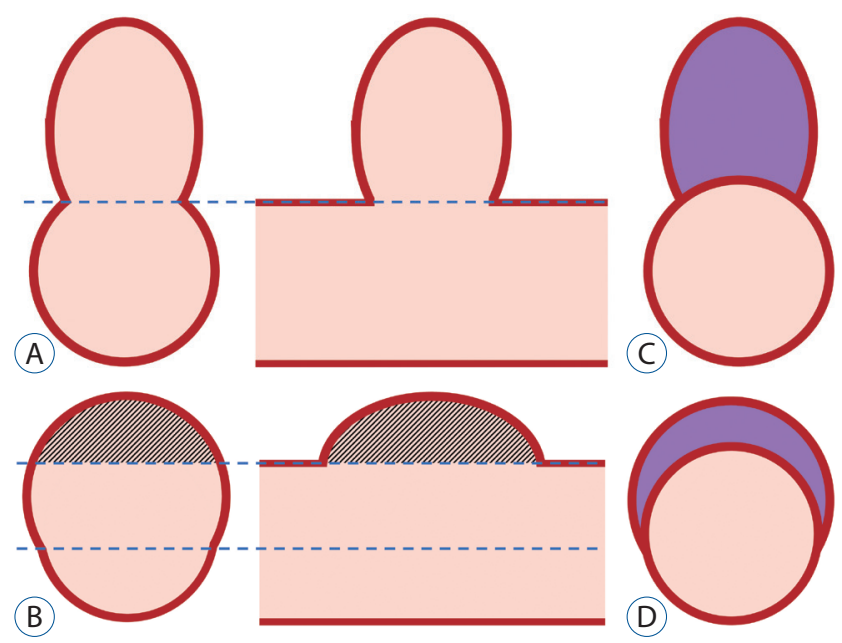

Fig. 1. Aneurysm volume of saccular and fusiform aneurysm. A : Classically, aneurysm volume can be defined as an outpouching lesion above the aneurysm neck in the saccular aneurysm. B : However, aneurysm neck may be frustrating in the fusiform aneurysm. C and D : Therefore, in this paper, the aneurysm volume is defined as the volume of the remaining part after removing the artery component.

neck is rather complicated in fusiform aneurysms and application of the same volume definition to both saccular and fusiform aneurysms may not apply (Fig. 1B). For this study, we defined aneurysm volume as the remnant volume after removing the normal arterial component, which can be applied to aneurysms of any shape or type (Fig. 1C and D). The data of 50 randomly selected aneurysms from our 3D digital subtraction angiography (DSA) Digital Imaging and Communications in Medicine (DICOM) database which were collected from March 2018 to June 2019 were used to compare and validate the different AVQ methods.

\section{Aneurysm volume measurement}

The basic idea of our novel universal 3D AVQ method is to differentiate the normal artery volume from the aneurysm prior to volume measurement (Fig. 2). Three steps are involved : data conversion, 3D model modification, and aneurysm volume measurement (Supplementary Video 1).

Conversion of DICOM data to a 3D model and model postprocessing

Open-source 3D Slicer software, version 4.10.2 (http:// www.slicer.org) and RadiAnt DICOM viewer software, version 5.0.0 (http://www.radiantviewer.com; Medixant, Poznań, Poland) were used to convert DICOM data into a 3D model ${ }^{10)}$. 


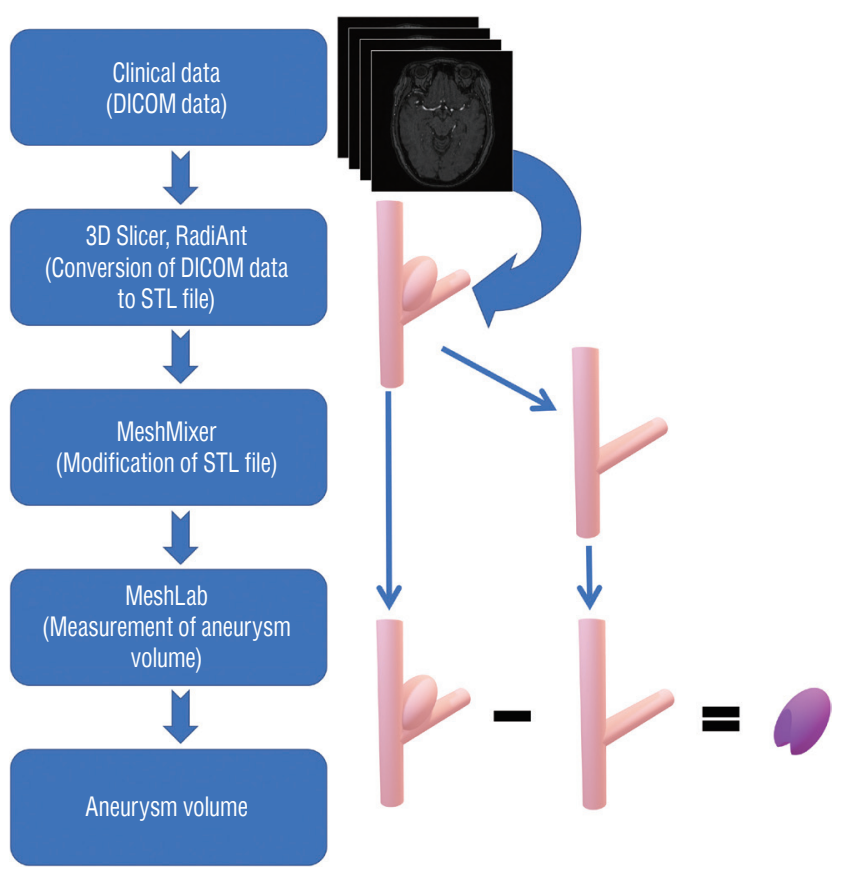

Fig. 2. The basic concept of the proposed novel aneurysm volume quantification method. Digital Imaging and Communications in Medicine data is converted to the 3-dimensional (3D) model and exported to a 3D STL file format. This 3D to stereolithography (STL) model is modified to reach the normal vasculature. The volume difference between 3D STL model with/without the aneurysm is defined as the aneurysm volume.

We then compared the 3D Slicer, RadiAnt, and ellipsoid AVQ methods using the 50 aneurysms from our DICOM database. In $3 \mathrm{D}$ Slicer, the vasculature was isolated by modifying the threshold tool. The threshold level was adjusted until the major cerebral blood vessels showed clear and distinct margins on axial, sagittal, and coronal images. After rendering the vascular 3D model, it was exported in stereolithography (STL) file format. In RadiAnt, the 3D model was generated with the $3 \mathrm{D}$ volume rendering tool, adjusted by controlling the window level, and also exported in STL file format. The segmentation of 3D models can be done in current step using 3D slicer and RadiAnt. But it can also be done in the next step, which is $3 \mathrm{D}$ STL model modification.

\section{D STL model modification}

Once STL files were exported, the 3D STL model was opened with Meshmixer software, version 3.5 (http://www. meshmixer.com; Autodesk, Inc., San Rafael, CA, USA). The aneurysm was digitally removed and converted to a normal arterial vascular feature by applying the edit and sculpt tool.
During the modification of a STL file, it is important not to make any modification of any other vessel components. Two 3D models of each patient were generated, one which included the aneurysm and one which excluded it and contained digitally reconstructed normal vasculature in its place.

\section{Aneurysm volume measurement}

The volumes of the two 3D models were measured with open-source Meshlab software, version 2016 (Visual Computing Lab, ISTI - CNR, Pisa, Italy). The difference between the two volumes was defined as the aneurysm volume.

\section{Aneurysm morphology parameters and the clas- sical ellipsoid method for AVQ}

Aneurysm height, neck size, and aspect ratio were measured as described by Dhar et al. ${ }^{7}$. The classical aneurysm volume measurement method that considers the aneurysm as an ellipsoid (ellipsoid method) was followed as described by Satoh et al. ${ }^{27)}$.

$$
\text { Aneurysm Volume }=\frac{4}{3} \pi\left(\frac{\text { height }}{2} \times \frac{\text { length }}{2} \times \frac{\text { width }}{2}\right)
$$

\section{Statistical analysis}

Statistical analysis was performed with SPSS software, version 25 (IBM, Inc., Armonk, NY, USA). Descriptive statistics of aneurysmal morphologic parameters are presented as means \pm standard deviation (SD). The agreement between AVQ methods was assessed with Bland-Altman plots using a $10 \%$ difference limit of acceptability with 3D Slicer data as the reference value ${ }^{3,6}$. Difference ratios in the Bland-Altman plots were converted to percentile values. AVQ methods were compared using the paired t-test. $p<0.05$ was considered significant. As the ellipsoid method is not applicable to fusiform aneurysms, data of four cases of fusiform aneurysms were discarded in comparing the intermethod agreement through the Bland-Altman plot.

\section{RESULTS}

The PubMed literature search identified 215 studies published between January 1997 and June 2019; 95 were not relevant for the purposes of this investigation and therefore excluded. Finally, 120 studies were reviewed and classified. 


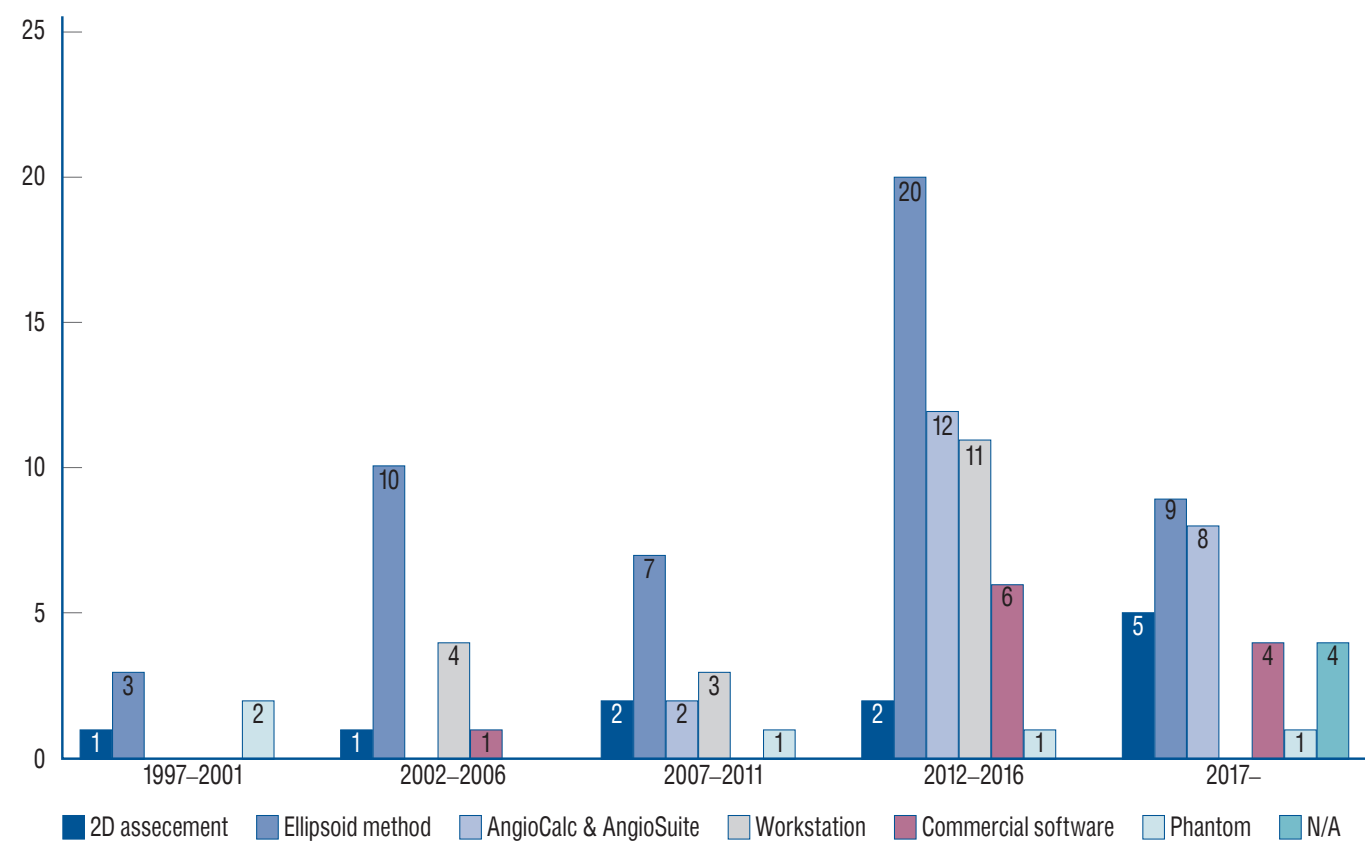

Fig. 3. The trend of aneurysm volume quantification (AVQ) methods. The ellipsoid method is still a major AVQ method although its limitations (approximately 60\%). AVQ method using the 3-dimensional model does not exceed one third in 5 year basis. N/A : not applicable.

Although AngioCalc and AngioSuite provide an interface by which users can manually input aneurysm volume, they calculate AVQ based on the ellipsoid method, as do several other AVQ methods. Hence, approximately $60 \%$ of all studies included in this investigation used the ellipsoid method; additionally, approximately $9 \%$ of the studies used 2D assessment and 25\% used a 3D model, which does not exceed one third on a 5 -year basis (Fig. 3 and Supplementary Data 1).

Volume of the 50 aneurysms randomly selected from our DICOM database were measured using 3D Slicer, Radiant DICOM viewer, and the ellipsoid method. Forty-six sacular aneurysms were included in comparison analysis of intermethod agreement; 16 of these were lobulated. The remaining four aneurysms were fusiform. Mean maximal aneurysm diameter and diameter of the aneurysm neck were approximately $6 \mathrm{~mm}$ and $4 \mathrm{~mm}$, respectively. Using 3D Slicer as a reference, the ratio of mean difference to mean volume obtained using RadiAnt and the ellipsoid method were $-1.21 \pm 7.46 \%$ and $4.04 \pm$ $30.54 \%$, respectively. Although the methods were used with aneurysms of many different shapes and configurations, the SDs in RadiAnt and 3D Slicer were very close. When comparing the ellipsoid method to 3D Slicer, the SD difference between methods increased in lobulated and fusiform aneurysms compared to non-lobulated aneurysms. The ratio of volume difference less than $10 \%$ between RadiAnt to 3D Slicer is almost twice of Ellipsoid method to 3D Slicer (Table 1). This implies that intermethod agreement is higher between RadiAnt and 3D Slicer than the ellipsoid method and 3D Slicer.

The Bland-Altman plots demonstrate the degree of agreement among the AVQ methods using 3D Slicer as the reference. The RadiAnt-3D Slicer plot shows that most mean volume differences were under $10 \%$. The difference in SD between RadiAnt and 3D Slicer was less than the difference between the ellipsoid method and 3D Slicer (Fig. 4). Note that the SD of RadiAnt and 3D Slicer is within 10\% of mean volume difference while the SD of the ellipsoid method and 3D Slicer is beyond $10 \%$ of mean volume difference. In the subanalysis based on aneurysm shape, the SD difference between RadiAnt and 3D Slicer was small. However, when comparing the difference between the ellipsoid method and 3D Slicer, SD tended to be larger in lobulated and fusiform aneurysms (Fig. 5). The SD of RadiAnt and 3D Slicer is within 10\% of mean volume difference while the SD of the ellipsoid method and 3D Slicer is beyond $10 \%$ of mean volume difference in both saccular and irregular shape of the aneurysm. Additional Bland-Altman plot of four cases of fusiform aneurysms is also demonstrated in Fig. 5. 
Table 1. Aneurysm morphologic parameters of 50 randomly selected aneurysms and statistical results

\begin{tabular}{|c|c|c|c|c|c|}
\hline & & $\begin{array}{c}\text { Aneurysm } \\
\text { charicteristic }\end{array}$ & 3D slicer & RadiAnt & Ellipsoid method \\
\hline Maximal diameter (mm), overall & & $6.03 \pm 2.11$ & & & \\
\hline Aneurysm neck (mm), overall & & $4.04 \pm 1.72$ & & & \\
\hline Aspect ratio, overall & & $1.22 \pm 0.54$ & & & \\
\hline \multicolumn{6}{|l|}{ Volume $\left(\mathrm{mm}^{3}\right)$} \\
\hline & Overall (50) & & $88.14 \pm 96.91$ & $90.35 \pm 100.64$ & $88.43 \pm 106.00$ \\
\hline & Saccular (46) & Non-lobulated (30) & $69.38 \pm 74.22$ & $71.95 \pm 77.36$ & $68.27 \pm 79.90$ \\
\hline & & Lobulated (16) & $127.45 \pm 129.24$ & $130.99 \pm 134.96$ & $127.66 \pm 144.99$ \\
\hline & Fusiform (4) & & $71.63 \pm 67.24$ & $65.82 \pm 59.42$ & N/A \\
\hline \multicolumn{6}{|l|}{ Mean volume difference ratio (\%) } \\
\hline & Overall (50) & & Reference & $-1.81 \pm 7.13$ & $5.80 \pm 30.22$ \\
\hline & Saccular (46) & Non-lobulated (30) & Reference & $-2.55 \pm 7.23$ & $4.91 \pm 17.60$ \\
\hline & & Lobulated (16) & Reference & $-0.42 \pm 6.97$ & $7.47 \pm 46.23$ \\
\hline & Fusiform (4) & & Reference & $5.68 \pm 8.82$ & N/A \\
\hline \multicolumn{6}{|c|}{ Mean volume difference within $10 \%$} \\
\hline & Overall (50) & & Reference & $78 \%$ & $38 \%$ \\
\hline & Saccular (46) & Non-lobulated (30) & Reference & $73 \%$ & $40 \%$ \\
\hline & & Lobulated (16) & Reference & $94 \%$ & $38 \%$ \\
\hline & Fusiform (4) & & Reference & $50 \%$ & N/A \\
\hline
\end{tabular}

Values are presented as mean \pm standard deviation or number. Statistical analysis of aneurysm morphologic parameters of 50 randomly selected aneurysms and statistical results. Note that the degree of agreement between 3D slicer and RadiAnt is higher than that of 3D slicer and ellipsoid method. 3D : 3-dimensional, N/A : not applicable
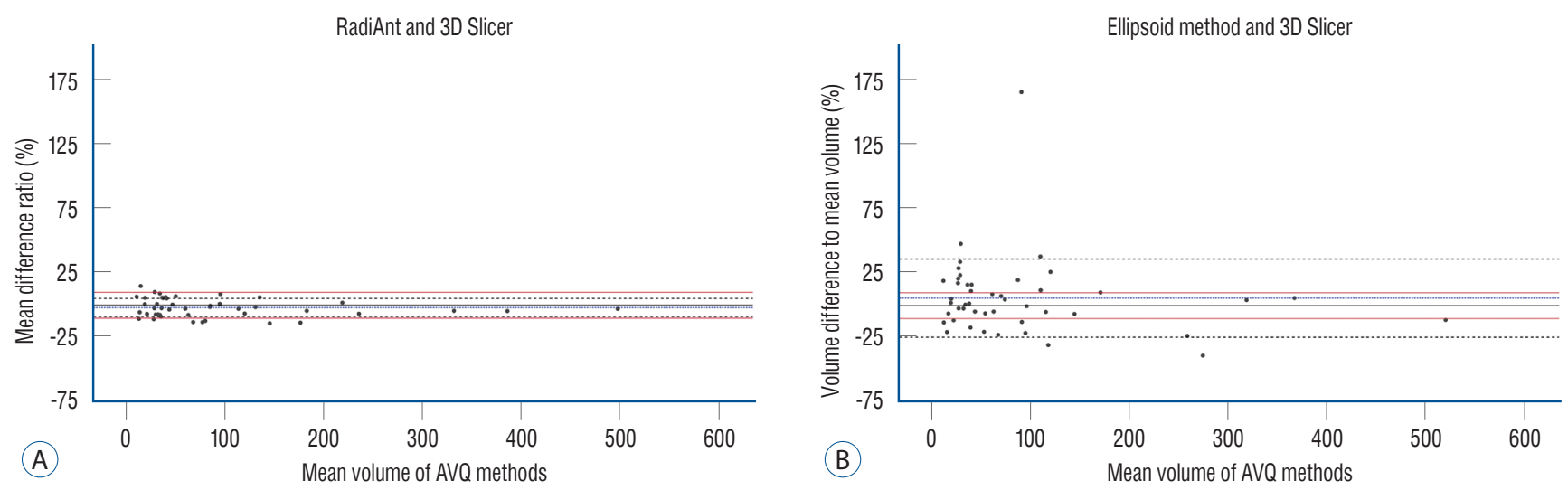

Fig. 4. A and B : Bland-Altman plots of RadiAnt-3-dimensional (3D) slicer and ellipsoid method-3D slicer. Standard deviation in Bland-Altman plots of RadiAnt-3D slicer is within 10\% of mean volume difference while standard deviation in Bland-Altman plots of ellipsoid method-3D slicer is beyond 10\% of mean volume difference. Red line : 10\% volume difference reference line, Black broken line : standard deviation, Blue broken line : mean volume difference to mean volume in percentile.

\section{DISCUSSION}

The concept of VER was introduced to represent the volume of embolized material (e.g., coils) versus aneurysm vol- ume. Although the term "packing density" is also widely used to represent the same concept, it refers to radiographic opacity inside an aneurysm visualized on 2D plain angiography. The widely used Raymond-Roy classification is based upon this 
RadiAnt and 3D Slicer

Aneurysm shape : saccular - non-lobulated

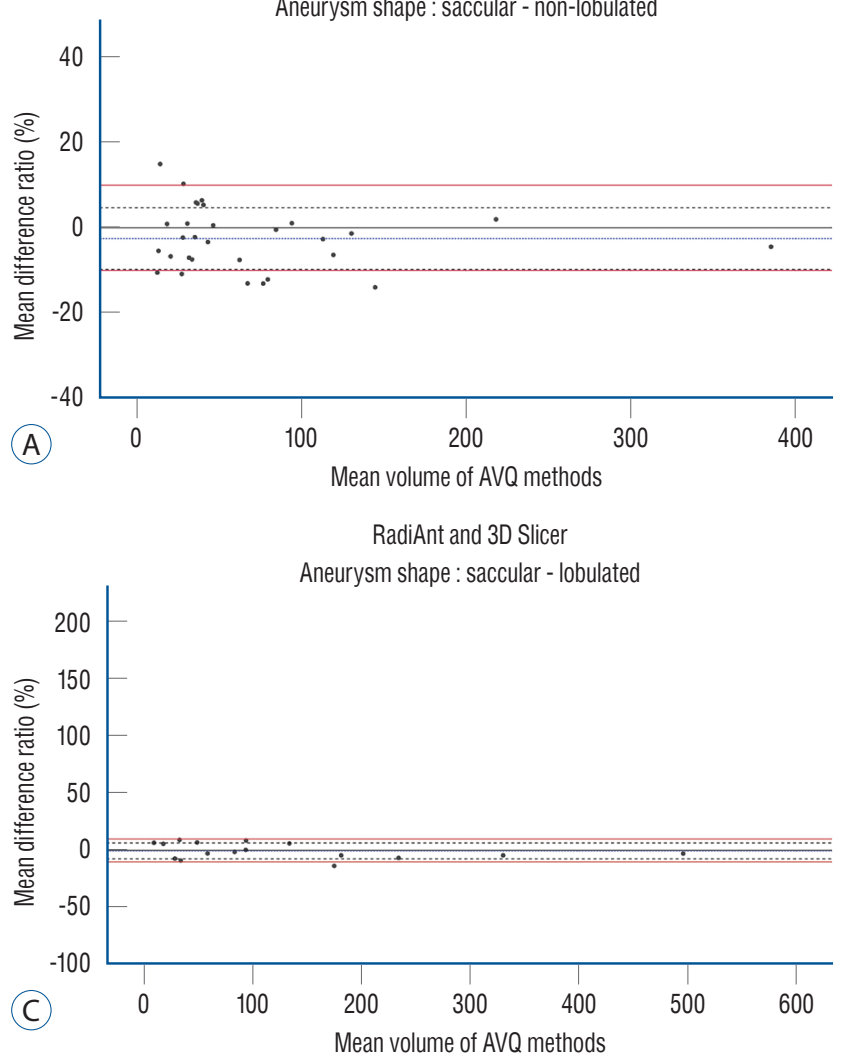

3D Slicer vs. RadiAnt

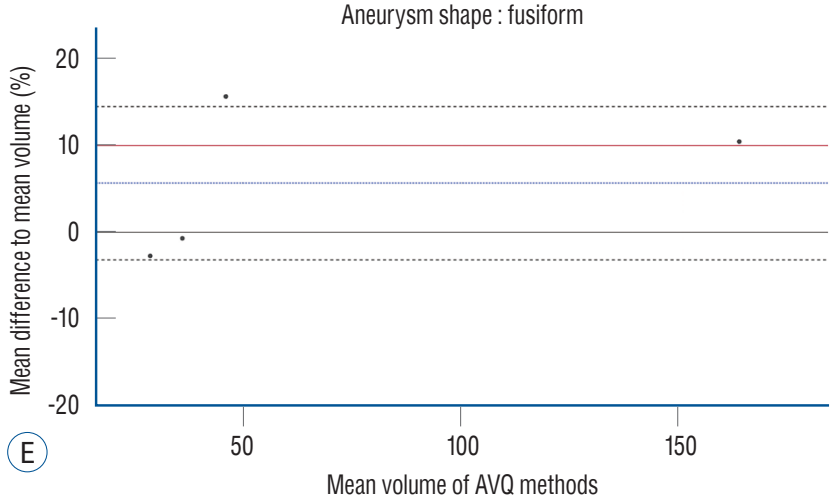

Ellipsoid method and 3D Slicer

Aneurysm shape : saccular - non-lobulated

(B)

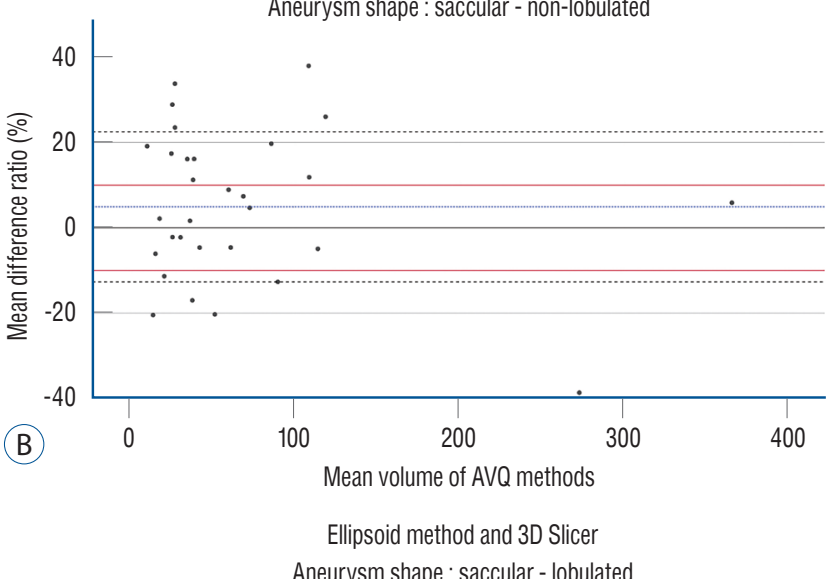

(D)

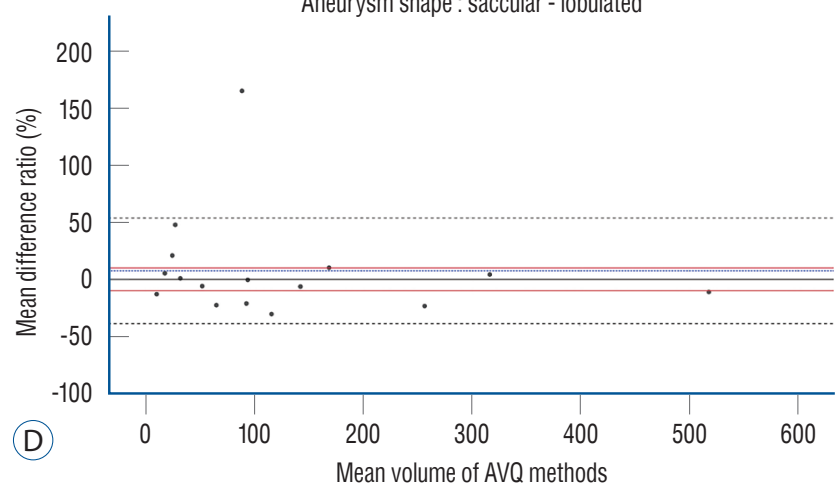

Fig. 5. Comparison of Bland-Altman plots by different aneurysm shapes. The standard deviation in Bland-Altman plots of RadiAnt-3-dimensional (3D) slicer is within $10 \%$ of mean volume difference in both non-lobulated and lobulated aneurysm shape (A and C). On the contrary, the standard deviation in Bland-Altman plots of ellipsoid method-3D slicer is beyond $10 \%$ of mean volume difference (B and D). Bland-Altman plots of RadiAnt-3D slicer in fusiform aneurysm is also demonstrated to show that current aneurysm volume quantification method is also applicable in fusiform aneurysm (E). Red line : reference line of $10 \%$ volume difference, Black broken line : standard deviation, Blue broken line : mean volume difference to mean volume in percentile.

concept $^{21,24,29,34)}$. It is relatively easy to calculate coil volume by assuming the coil as a cylinder. Traditionally, AVQ has been calculated by considering the aneurysm as an ellipsoid ${ }^{27)}$. However, the accuracy of this method has been questioned and debated ${ }^{26,32)}$. Many authors have pointed out that the el- lipsoid method may underestimate or miscalculate the volume, particularly in wide-neck and lobulated aneurysms. Nevertheless, it has been widely used due to its simplicity. Low VER and remnant volume have been associated with aneurysm recurrence in previous studies ${ }^{16,17,22,25,28,33)}$. Although 
many studies have been mentioning VER, the majority still use the ellipsoid method for AVQ (Fig. 3 and Supplementary Data 1). To achieve better accuracy, a method using 3D rendered data for AVQ has been recommended.

There are several issues to point out regarding determination of aneurysm volume. First, aneurysm volume can only be measured indirectly; it cannot be measured directly in vivo. Therefore, DICOM data must be used to determine aneurysm volume. However, different imaging modalities and patient condition (e.g., blood pressure, pulse, etc.) may affect the indirect measurement of aneurysm volume. Second, aneurysm volume is usually defined as the volume of the abnormal outpouching vascular lesion above the aneurysm neck. However, in fusiform aneurysms, determination of the aneurysm neck is rather complicated, and applying the same definition of volume to both saccular and fusiform aneurysms is rather difficult. Therefore, we elected to define aneurysm volume as the remnant volume after removing the normal arterial volume. This definition can be applied to aneurysms of any shape.

A universal method for AVQ should fulfill several conditions. First, it should have a low barrier to entry for general use; the method should be easy to apply and interpret and cost little. Second, it should be able to be performed expeditiously; if it requires $>30$ minutes to calculate, the method may not be accepted for widespread use. Third, it should be able to be used with various imaging modalities, such as computed tomography angiography (CTA), magnetic resonance angiography (MRA), and DSA. Finally, the method should be accurate and reproducible and able to be used for any aneurysm shape or type.

For the universal AVQ method proposed here, the first step is to convert DICOM data into a 3D model. Since 3D Slicer has been commonly used in various medical and bioengineering fields, we elected to use it as the reference method $\mathrm{d}^{1,11,12,20,31)}$. Although 3D Slicer has been previously used to define cerebral vascular anatomy and obtain vascular parameters, to our knowledge, it has not been used to measure aneurysm volume $^{4,13-15,18,19)}$. After the 3D model is generated in 3D Slicer, it is possible to measure the aneurysm volume by segmentation. The segmentation process in 3D Slicer is easily implemented for saccular aneurysms, but not for fusiform aneurysms or aneurysms of a curved vessel. Therefore, we exported the 3D model in STL file format and sculped it with Meshmixer, software which was designed for 3D printing of STL files. Since Meshmixer provides various tools to edit 3D STL files, it can be used for aneurysms of any shape. However, volume cannot be measured with Meshmixer; this was accomplished using Meshlab. Although three separate programs are required, all are free or open-source software available on the internet.

During the conduction of this study, we found a considerable learning curve with using 3D Slicer : a 3D STL model could be generated in $<3$ minutes by experienced users but beginners required almost 40 . However, RadiAnt can also export 3D rendered data in STL file format. Its interface is very intuitive and easy to learn and it requires fewer processing steps: experienced users and beginners alike required $<5$ minutes to generate a 3D STL model. We validated high intermethod agreement between 3D Slicer and RadiAnt. In addition, both 3D Slicer and RadiAnt support various imaging modalities including CTA, MRA, and 3D DSA. Pros and cons of each software and AVQs are presented in Table 2.

The Bland-Altman plot shows the SD of volume difference in AVQ methods performed with 3D STL models in within $10 \%$ of mean volume difference which represent a high degree

Table 2. Comparison of pricing, difficulty and available function between software and aneurysm volume quantification method

\begin{tabular}{|c|c|c|c|c|c|c|c|c|}
\hline & 3D Slicer & RaniAnt & MeshMixer & MeshLab & Workstation & $\begin{array}{l}\text { Ansys } \\
\text { system }\end{array}$ & $\begin{array}{c}\text { CAD } \\
\text { software }\end{array}$ & $\begin{array}{l}\text { Ellipsoid } \\
\text { method }\end{array}$ \\
\hline Pricing & Open source & Up to $110 \$^{*}$ & Free & Open source & High & High & High & Free \\
\hline Overall level of difficulty & Medium & Low & $\begin{array}{l}\text { Low to } \\
\text { medium }\end{array}$ & Low & High & High & High & Low \\
\hline Segmentation difficulty & Medium & Low & $\begin{array}{l}\text { Low to } \\
\text { medium }\end{array}$ & Low & Medium & High & High & Low \\
\hline Volume measurement & Available & Not available & Not available & Available & Available & Available & Available & Available \\
\hline Accuracy & High & High & High & High & High & High & High & Low \\
\hline
\end{tabular}

*Free trial version and various subscription plan is provided. 3D : 3-dimensional, CAD : computer-aided design 
of intermethod agreement (Figs. 4 and 5). The specific software used to convert DICOM data into a 3D STL model does not appear to affect the result, even in aneurysms of various shapes, because the DICOM data itself functions as a representative depiction of the vasculature. However, the ellipsoid method showed a lower degree of agreement than 3D STL which the SD is beyond $10 \%$ of mean volume difference which represents the result of low intermethod agreement (Figs. 4 and 5). The intermethod agreement was even worse in multilobulated and fusiform aneurysms (Fig. 5). This indicates that AVQ with a 3D STL model is more reliable in any type of aneurysm than the ellipsoid method. Therefore, AVQ using a 3D STL model has the potential to become a universal method in future studies investigating the correlation between aneurysm recurrence and VER.

We encountered a similar AVQ idea in a study by Costalat et al. ${ }^{6}$ The authors used software that automatically calculates the erased volume from a 3D rendered model: the aneurysm is erased until a normal artery form is reached. Although this resembles the basic concept of previous studies, their study was the only one using this method according to our PubMed search. The reproducibility and accuracy of the method in Costalat et al. ${ }^{6}$ were high, but its use would be limited as it requires special proprietary software. Although most angiography suite workstations provide various methods of AVQ, if the aneurysm is fusiform or contains multiple lobes, it may not be accurately detected. In addition, CAD and CFD software have also been used for AVQ, but their high cost and level of skill required heighten the entry barrier for widespread general use (Table 2).

An additional advantage of using our proposed novel AVQ method is derived from the data conversion process which exports vasculature data into a 3D STL model. This STL file format was developed by 3D Systems (Rock Hill, SC, USA) to store 3D geographic data in the 1980s and has been used in various fields including CAD and $3 \mathrm{D}$ printing. Therefore, a 3D STL model can be exported to CFD software to investigate intravascular fluid dynamics and to $3 \mathrm{D}$ printing software to generate phantom models. Use of this novel AVQ method may lead to improved understanding of aneurysmal fluid dynamics and inspire new clinical and scientific advancement.

Recently, the interest in endovascular treatment of intracranial aneurysm has shifted to flow diverters. For this reason, the interest of VER in endovascular coiling seems to be de- creased. However, aneurysm coiling is still the mainstream in endovascular treatment and VER is a crucial factor in recurrence of coiled aneurysm. For objective calculation of VER, reasonable method for AVQ is essential. Furthermore, to understand the nature of the aneurysm and endovascular coiling, quantification and interpretation of the aneurysm and surrounding vascular anatomy is crucial, especially in the era of CFD. Therefore, we assure that refining the definition of the aneurysm and developing an AVQ for various shapes of aneurysms can give us more information in understanding the nature of intracranial aneurysms.

\section{CONCLUSION}

Even in the era of 3D angiography, most clinical and scientific studies still rely on the ellipsoid method for AVQ despite its problems with accuracy and incompatibility for use with fusiform and irregularly shaped aneurysms. To overcome these problems, we propose a novel universal method for AVQ that utilizes user-friendly open source 3D STL model editing software that is available on the internet. We believe this novel method will be helpful in clinical practice and in future scientific studies.

\section{CONFLICTS OF INTEREST}

No potential conflict of interest relevant to this article was reported.

\section{INFORMED CONSENT}

This type of study does not require informed consent.

\section{AUTHOR CONTRIBUTIONS}

\author{
Conceptualization : JC \\ Data curation : JC \\ Formal analysis : JC \\ Methodology : JC \\ Project administration : JC
}




\author{
Visualization : JC, JHK \\ Writing - original draft : JC, JHK \\ Writing - review \& editing : JC, JHK
}

\section{ORCID}

$\begin{array}{ll}\text { Jaewoo Chung } & \text { https://orcid.org/0000-0002-3512-6610 } \\ \text { Jung Ho Ko } & \text { https://orcid.org/0000-0002-1185-4381 }\end{array}$

\section{- Acknowledgements}

We would like to express my sincere thanks to Dr. Sang Kwon Chung, Professor Emeritus of Department of Mathematics Education, Seoul National University, for his invaluable comments and suggestions on dealing with the mathematical interpretation of CFD simulations.

\section{- Supplementary materials}

The online-only data supplement is available with this article at https://doi.org/10.3340/jkns.2020.0255.

\section{References}

1. Abi-Aad KR, Aoun RJN, Rahme RJ, Ward JD, Kniss J, Kwasny MJ, et al. : New generation hydrogel endovascular aneurysm treatment trial (HEAT): a study protocol for a multicenter randomized controlled trial. Neuroradiology 60 : 1075-1084, 2018

2. Bescós JO, Slob MJ, Slump CH, Sluzewski M, van Rooij WJ : Volume measurement of intracranial aneurysms from 3D rotational angiography: improvement of accuracy by gradient edge detection. AJNR Am J Neuroradiol 26 : 2569-2572, 2005

3. Bland JM, Altman DG : Statistical methods for assessing agreement between two methods of clinical measurement. Lancet $1: 307-310,1986$

4. Can A, Ho AL, Emmer BJ, Dammers R, Dirven CM, Du R : Association between vascular anatomy and posterior communicating artery aneurysms. World Neurosurg 84 : 1251-1255, 2015

5. Chan SH, Wong KS, Woo YM, Chan KY, Leung KM : Volume measurement of the intracranial aneurysm: a discussion and comparison of the alternatives to manual segmentation. J Cerebrovasc Endovasc Neurosurg $16: 358-363,2014$

6. Costalat V, Maldonado IL, Strauss O, Bonafé A : Toward accurate volumetry of brain aneurysms: combination of an algorithm for automatic thresholding with a 3D eraser tool. J Neurosci Methods 198 : 294300,2011
7. Dhar S, Tremmel M, Mocco J, Kim M, Yamamoto J, Siddiqui AH, et al. : Morphology parameters for intracranial aneurysm rupture risk assessment. Neurosurgery 63 : 185-196; discussion 196-197, 2008

8. Escobar-de la Garma VH, Zenteno M, Padilla-Vázquez F, San-Juan D, Cerón-Morales A : Comparative analysis of aneurysm volume by different methods based on angiography and computed tomography angiography. Neurosurg Rev 41 : 1013-1019, 2018

9. Fanning NF, O'Dwyer HM, Bowden JA, Brennan PR, Thornton J : Accuracy of voxel-based and algebraic formula-based methods in quantifying cerebral aneurysm volume by 3D-rotational digital subtraction angiography. An in-vitro and in-vivo study. Interv Neuroradiol 11 : 35-40, 2005

10. Fedorov A, Beichel R, Kalpathy-Cramer J, Finet J, Fillion-Robin JC, Pujol $S$, et al. : 3D slicer as an image computing platform for the quantitative imaging network. Magn Reson Imaging 30 : 1323-1341, 2012

11. Ferroli P, Tringali G, Acerbi F, Schiariti M, Broggi M, Aquino D, et al. : Advanced 3-dimensional planning in neurosurgery. Neurosurgery 72 Suppl 1 : 54-62, 2013

12. Han Q, Zhao X, Wang C, Chen B, Wang X, Zhang Z, et al. : Individualized reconstruction for severe periprosthetic fractures around the tumor prosthesis of knee under assistance of 3D printing technology: a case report. Medicine (Baltimore) 97 : e12726, 2018

13. Ho AL, Mouminah A, Du R : Posterior cerebral artery angle and the rupture of basilar tip aneurysms. PLoS One 9 : e110946, 2014

14. Jiang H, Shen J, Weng YX, Pan JW, Yu JB, Wan ZA, et al. : Morphology parameters for mirror posterior communicating artery aneurysm rupture risk assessment. Neurol Med Chir (Tokyo) 55 : 498-504, 2015

15. Jiang H, Weng YX, Zhu Y, Shen J, Pan JW, Zhan RY : Patient and aneurysm characteristics associated with rupture risk of multiple intracranial aneurysms in the anterior circulation system. Acta Neurochir (Wien) 158 : 1367-1375, 2016

16. Kawanabe $Y$, Sadato A, Taki W, Hashimoto $N$ : Endovascular occlusion of intracranial aneurysms with Guglielmi detachable coils: correlation between coil packing density and coil compaction. Acta Neurochir (Wien) $143:$ 451-455, 2001

17. Leng B, Zheng Y, Ren J, Xu Q, Tian Y, Xu F : Endovascular treatment of intracranial aneurysms with detachable coils: correlation between aneurysm volume, packing, and angiographic recurrence. J Neurointerv Surg 6 : 595-599, 2014

18. Lin N, Ho A, Charoenvimolphan N, Frerichs KU, Day AL, Du R : Analysis of morphological parameters to differentiate rupture status in anterior communicating artery aneurysms. PLoS One 8 : e79635, 2013

19. Lin N, Ho A, Gross BA, Pieper S, Frerichs KU, Day AL, et al. : Differences in simple morphological variables in ruptured and unruptured middle cerebral artery aneurysms. J Neurosurg 117 : 913-919, 2012

20. Munkvold BKR, Bø HK, Jakola AS, Reinertsen I, Berntsen EM, Unsgård G, et al. : Tumor volume assessment in low-grade gliomas: a comparison of preoperative magnetic resonance imaging to coregistered intraoperative 3-dimensional ultrasound recordings. Neurosurgery 83 : 288-296, 2018

21. Murayama Y, Viñuela F, Ishii A, Nien YL, Yuki I, Duckwiler $G$, et al. : Ini- 
tial clinical experience with matrix detachable coils for the treatment of intracranial aneurysms. J Neurosurg 105 : 192-199, 2006

22. Otani T, Nakamura M, Fujinaka T, Hirata M, Kuroda J, Shibano K, et al. : Computational fluid dynamics of blood flow in coil-embolized aneurysms: effect of packing density on flow stagnation in an idealized geometry. Med Biol Eng Comput 51 : 901-910, 2013

23. Piotin M, Daghman B, Mounayer C, Spelle L, Moret J : Ellipsoid approximation versus $3 \mathrm{D}$ rotational angiography in the volumetric assessment of intracranial aneurysms. AJNR Am J Neuroradiol 27 : 839-842, 2006

24. Reul J, Spetzger U, Weis J, Sure U, Gilsbach JM, Thron A : Endovascular occlusion of experimental aneurysms with detachable coils: influence of packing density and perioperative anticoagulation. Neurosurgery 41 : 1160-1165; discussion 1165-1168, 1997

25. Sadato A, Hayakawa M, Adachi K, Nakahara I, Hirose Y : Large residual volume, not low packing density, is the most influential risk factor for recanalization after coil embolization of cerebral aneurysms. PLoS One 11 : e0155062, 2016

26. Sadato A, Hayakawa M, Tanaka $T$, Hirose $Y$ : Comparison of cerebral aneurysm volumes as determined by digitally measured $3 \mathrm{D}$ rotational angiography and approximation from three diameters. Interv Neuroradiol $17:$ 154-158, 2011

27. Satoh $K$, Matsubara $S$, Hondoh $H$, Nagahiro $S$ : Intracranial aneurysm embolization using interlocking detachable coils. Correlation between volume embolization rate and coil compaction. Interv Neuroradiol 3 Suppl 2 : 125-128, 1997
28. Sluzewski M, van Rooij WJ, Slob MJ, Bescós JO, Slump CH, Wijnalda D : Relation between aneurysm volume, packing, and compaction in 145 cerebral aneurysms treated with coils. Radiology 231 : 653-658, 2004

29. Sokolowski JD, Ilyas A, Buell TJ, Taylor DG, Chen CJ, Ding D, et al. : SMART coils for intracranial aneurysm embolization: follow-up outcomes. J Clin Neurosci 59 : 93-97, 2019

30. Stapleton CJ, Torok CM, Rabinov JD, Walcott BP, Mascitelli JR, LeslieMazwi TM, et al. : Validation of the modified Raymond-Roy classification for intracranial aneurysms treated with coil embolization. J Neurointerv Surg 8 : 927-933, 2016

31. Strik HM, Borchert $H$, Fels $C$, Knauth $M$, Rienhoff $O$, Bähr $M$, et al. : Three-dimensional reconstruction and volumetry of intracranial haemorrhage and its mass effect. Neuroradiology 47 : 417-424, 2005

32. Takao H, Ishibashi T, Saguchi T, Arakawa H, Ebara M, Irie K, et al. : Validation and initial application of a semiautomatic aneurysm measurement software: a tool for assessing volumetric packing attenuation. AJNR Am J Neuroradiol 35 : 721-726, 2014

33. Wakhloo AK, Gounis MJ, Sandhu JS, Akkawi N, Schenck AE, Linfante I : Complex-shaped platinum coils for brain aneurysms: higher packing density, improved biomechanical stability, and midterm angiographic outcome. AJNR Am J Neuroradiol 28 : 1395-1400, 2007

34. Zhao R, Shen J, Huang QH, Nie JH, Xu Y, Hong B, et al. : Endovascular treatment of ruptured tiny, wide-necked posterior communicating artery aneurysms using a modified stent-assisted coiling technique. J Clin Neurosci 20 : 1377-1381, 2013 